\title{
A set of miRNAs participates in the cellular senescence program in human diploid fibroblasts
}

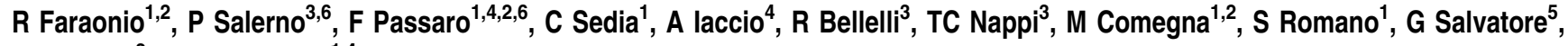 \\ M Santoro ${ }^{3}$ and F Cimino ${ }^{\star, 1,4}$
}

Here we show that replicative senescence in normal human diploid IMR90 fibroblasts is accompanied by altered expression of a set of microRNAs (miRNAs) (senescence-associated miRNAs), with 14 and 10 miRNAs being either up or downregulated ( $>$ 2-fold), respectively, in senescent with respect to young cells. The expression of most of these miRNAs was also deregulated upon senescence induced by DNA damage (etoposide) or mild oxidative stress (diethylmaleate). Four downregulated miRNAs were part of miRNA family-17, recently associated to human cell and tissue aging. Moreover, eight upregulated and six downregulated miRNAs mapped in specific chromosomal clusters, suggesting common transcriptional regulation. Upon adoptive overexpression, seven upregulated miRNAs induced the formation of senescence-associated heterochromatin foci and senescence-associated $\beta$-galactosidase staining $(P<0.05)$, which was accompanied, in the case of five of them, by reduced cell proliferation. Finally, miR-210, miR-376a*, miR-486-5p, miR-494, and miR-542-5p induced double-strand DNA breaks and reactive oxygen species accumulation in transfected cells. In conclusion, we have identified a set of human miRNAs induced during replicative and chemically induced senescence that are able to foster the senescent phenotype by prompting DNA damage.

Cell Death and Differentiation (2012) 19, 713-721; doi:10.1038/cdd.2011.143; published online 4 November 2011

Replicative or cellular senescence, a state of irreversible arrest of cell division, was first described in cultures of human fibroblasts. ${ }^{1}$ Since then, replicative senescence has been described in various mammalian cells. ${ }^{2}$ The mechanisms underlying senescence include telomere shortening, upregulation of the CDKN1A (p21WAF1) and CDKN2A (p16INK4a and p14ARF) loci, and accumulation of DNA damage. ${ }^{3}$ Telomeres become progressively shorter at every round of cell division and this leads to critically short telomere length sensed as double-strand DNA breaks. ${ }^{4}$ DNA damage and DNA-damage response (DDR) could be common events to cellular senescence programs initiated by telomere dysfunction and aberrant oncogene activation. ${ }^{5}$

Senescent cells are marked by lack of DNA replication; expression of senescence-associated $\beta$-galactosidase (SA- $\beta$-gal); accumulation of discrete nuclear foci that are termed senescence-associated heterochromatin foci (SAHFs); and senescence-associated DNA-damage foci (SDFs). SAHFs are detected by preferential binding of DNA dyes, such as 4',6-diamidino-2-phenylindole (DAPI), and the presence of certain heterochromatin-associated histone modifications (trimethyl-Lys9 Histone H3). SDFs are nuclear foci containing proteins that are associated to DNA damage
(Ser139-phosphorylated histone $\mathrm{H} 2 \mathrm{AX}-\gamma-\mathrm{H} 2 \mathrm{AX}$ - and p53binding protein-1-53BP1). ${ }^{6}$

Senescent cells show striking changes in gene expression, including upregulation of cell-cycle inhibitors (p21WAF1 and p16INK4a) and secreted proteins involved in microenvironment remodeling (IL-6), ${ }^{7}$ and downregulation of genes that facilitate cell-cycle progression (c-FOS, cyclin-A, cyclin-B, $\mathrm{PCNA})^{8}$ or that are involved in cell-cycle execution (FOXM1, UBE2C, TYMS). ${ }^{9}$ Mechanisms underlying the gene expression program that is associated to senescence are still poorly understood.

MicroRNAs (miRNAs) are short (20-24 nt) non-coding RNAs that are involved in post-transcriptional regulation of gene expression. miRNAs are transcribed as part of primary transcripts (pri-miRNAs). The pri-miRNA is cleaved by the Drosha ribonuclease-III to produce an approximately 70-nt stem-loop precursor miRNA (pre-miRNA), which is further cleaved by the cytoplasmic Dicer ribonuclease to generate mature miRNA and antisense miRNA star (miRNA*) products. The mature miRNA is incorporated into an RNA-induced silencing complex (RISC), which recognizes target mRNAs and most commonly results in translational inhibition or destabilization of the target mRNA. ${ }^{10}$

\footnotetext{
${ }^{1}$ Dipartimento di Biochimica e Biotecnologie Mediche, Università di Napoli Federico II, Napoli, Italy; ${ }^{2}$ CEINGE-Biotecnologie Avanzate s.c. a r.l, Napoli, Italy; ${ }^{3}$ Istituto di Endocrinologia ed Oncologia Sperimentale (IEOS), C.N.R. c/o Dipartimento di Biologia e Patologia Cellulare e Molecolare, Università di Napoli Federico II, Napoli, Italy; ${ }^{4}$ IRCCS Fondazione SDN, Napoli, Italy and ${ }^{5}$ Dipartimento di Studi delle Istituzioni e dei Sistemi Territoriali, Università Parthenope, Napoli, Italy

*Corresponding author: F Cimino, Dipartimento di Biochimica e Biotecnologie Mediche, Università di Napoli Federico II, via S Pansini 5, Napoli 80131, Italy.

Tel: + 39081746 2107; Fax: + 39081746 3650; E-mail: cimino@dbbm.unina.it

${ }^{6}$ These authors contributed equally to this work.

Keywords: microRNA; cellular senescence; gene expression; DNA damage

Abbreviations: CDK, cyclin-dependent kinase; RISC, RNA-induced silencing microprocessor complex; SAHFs, senescence-associated heterochromatin foci; SDFs, senescence-associated DNA-damage foci; SA- $\beta$-gal, senescence-associated $\beta$-galactosidase; ATCC, American Type Culture Collection; DMEM, Dulbecco's modified Eagle's medium; PDL, population doubling level; PBS, phosphate-buffered saline; DMSO, dimethyl sulfoxide; DEM, diethylmaleate; BrdU, 5-bromo-2deoxyuridine; DAPI, 4',6-diamidino-2-phenylindole; DDR, DNA-damage response; DHE, dihydroethidium; mTOR, mammalian target of rapamycin

Received 21.3.11; revised 27.9.11; accepted 27.9.11; Edited by M Blagosklonny; published online 04.11.11
} 
Being potent regulators of gene expression, we hypothesized that miRNAs were involved in the gene expression program associated to cellular senescence. This hypothesis was supported by recent results obtained by Hackl et al. ${ }^{11}$ showing that miRNAs of family 17 were downregulated in aged tissues and cells. Moreover, senescence in human lung (WI-38) fibroblasts was accompanied by upregulation of miR-152, miR-410, miR-431, and miR-493, and downregulation of miR-155, miR-20a, miR-25, and miR-15a; knockdown of miR-155 or miR-20a enhanced IR-induced senescence. ${ }^{12,13}$

In this study, we profiled miRNA expression in human senescent fibroblasts (IMR90). We identified 24 miRNAs that were either up- or downregulated in senescent cells. Upon adoptive overexpression, most of the upregulated miRNAs reduced DNA synthesis rate, and induced SA- $\beta$-gal staining and SAHFs. Five of them induced a robust DNA damage that likely sustains the senescent phenotype.

\section{Results}

Altered expression of a set of miRNAs accompanies replicative senescence in IMR90 fibroblasts. At PDL58, more than $70 \%$ IMR90 cells stained positively for SA- $\beta$-gal, whereas less than $1 \%$ of PDL33 cells stained positively (Supplementary Figure 1). Moreover, PDL58 cells had an expression profile consistent with a senescent phenotype, expressing about three-fold higher levels of p21WAF1 and p16INK4a proteins; higher levels of IL6 mRNA; and reduced levels of cell-cycle-related mRNAs such as cyclin-A, thymidylate synthase (TYMS), cyclin-selective ubiquitin carrier protein (UBE2C), and forkhead box-M1 (FOXM1) with respect to PDL33 cells (Supplementary Figure 1). We profiled miRNA expression in senescent PDL58 with respect to PDL33 IMR90 cells by qRT-PCR. A total of 148 miRNAs were detected $\left(C_{\mathrm{t}}<39\right.$ cycles) in at least one of the two samples; 18 miRNAs were upregulated and 51 were downregulated (>2-fold) in senescent versus young cells (Supplementary Table 1).

To validate these results, independent preparations of PDL58 and PDL33 IMR90 cells were obtained and the expression of all the highest ranked 18 up- and 14 downregulated miRNAs was investigated in triplicate by qRT-PCR. Results are reported in Table 1. Fourteen (fold changes: 2.7-8.9) and 10 (fold changes: 0.42-0.13) miRNAs were significantly $(P<0.001)$ up- or downregulated in PDL58 versus PDL33 cells, respectively (Table 1 ).

These findings indicate that senescence of IMR90 is associated to altered expression of a set of miRNAs (hereafter referred to as 'senescence-associated miRNAs').

DNA damage and oxidative stress deregulate the expression of senescence-associated miRNAs. Treatment with etoposide, ${ }^{15}$ a topoisomerase inhibitor used to induce DNA damage, or DEM, ${ }^{9}$ a glutathione depletor able to cause a mild oxidative stress, were applied to chemically induce cellular senescence. When IMR90 cells were exposed to DEM $(100 \mu \mathrm{M})$ for 10 days or treated with etoposide $(20 \mu \mathrm{M})$ for $24 \mathrm{~h}$ and further cultured for 11 days, 62 and $80 \%$ SA- $\beta$-gal-positive IMR90 cells, respectively, were detected. This was accompanied by gene expression changes consistent with a senescent phenotype

Table 1 Expression change of senescence-associated miRNAs in IMR90 cells

\begin{tabular}{|c|c|c|c|c|c|}
\hline miRNA & $\begin{array}{l}\text { Chromosomal } \\
\text { location }^{\mathrm{a}}\end{array}$ & Family $^{a}$ & $\begin{array}{l}\text { Fold changes } \\
\text { (PDL58/PDL33) }\end{array}$ & S.D. & $P$-value \\
\hline \multicolumn{6}{|l|}{ Upregulated } \\
\hline miR-486-5p & $8 p 11.21$ & 486 & 8.9 & 0.009 & $<0.0001$ \\
\hline miR-210 & $11 \mathrm{p} 15.5$ & 210 & 7.9 & 0.9 & $<0.0001$ \\
\hline miR-30e-5p & $1 \mathrm{p} 34.2$ & 30 & 6.5 & 0.9 & $<0.0001$ \\
\hline miR-376a* & $14 q 32.31$ & 368 & 5.9 & 0.7 & 0.0001 \\
\hline $\operatorname{miR}-126^{*}$ & $9 q 34.3$ & 126 & 5.3 & 1.4 & $<0.0001$ \\
\hline miR-494 & $14 q 32.31$ & 154 & 4.5 & 1.6 & $<0.0001$ \\
\hline miR-379 & $14 q 32.31$ & 379 & 4.4 & 1.1 & $<0.0001$ \\
\hline miR-654 & $14 q 32.31$ & 654 & 4.3 & 1.4 & $<0.0001$ \\
\hline miR-542-5p & Xq26.3 & 542 & 3.8 & 1 & $<0.0001$ \\
\hline miR-23b & $9 q 22.32$ & 23 & 3.7 & 0.6 & 0.0022 \\
\hline miR-134 & $14 q 32.31$ & 134 & 3.4 & 1 & $<0.0001$ \\
\hline miR-369-3p & $14 q 32.31$ & 154 & 3.3 & 0.4 & $<0.0001$ \\
\hline miR-656 & $14 \mathrm{q} 32.31$ & 154 & 3.3 & 1 & $<0.0001$ \\
\hline miR-485-5p & $14 q 32.31$ & 485 & 2.7 & 0.5 & $<0.0001$ \\
\hline \multicolumn{6}{|l|}{ Downregulated } \\
\hline miR-20a & $13 q 31.3$ & 17 & 0.13 & 0.01 & 0.0002 \\
\hline miR-155 & $21 \mathrm{q} 21.3$ & 155 & 0.14 & 0.04 & 0.0034 \\
\hline miR-17-5p & $13 q 31.3$ & 17 & 0.15 & 0.02 & 0.0060 \\
\hline miR-199b-5p & $9 q 34.11$ & 199 & 0.21 & 0.09 & 0.0002 \\
\hline miR-15b & $3 q 25.33$ & 15 & 0.23 & 0.16 & 0.0008 \\
\hline $\operatorname{miR}-92^{b}$ & 13q31.3; Xq26.2 & 25 & 0.27 & 0.04 & 0.0001 \\
\hline miR-296-5p & $20 q 13.32$ & 296 & 0.27 & 0.2 & 0.0061 \\
\hline $\operatorname{miR}-19 b^{c}$ & $13 q$ 31.3; Xq26.2 & 19 & 0.28 & 0.1 & 0.0016 \\
\hline miR-93 & $7 q 22.1$ & 17 & 0.35 & 0.04 & $<0.0001$ \\
\hline $\mathrm{miR}-106 \mathrm{~b}$ & $7 q 22.1$ & 17 & 0.42 & 0.17 & 0.01 \\
\hline
\end{tabular}

${ }^{\mathrm{a}}$ The information were obtained from miRBase (http://www.mirbase.org/). ${ }^{\mathrm{b}}$ miR-92 includes two identical sequences (miR-92a-1 and miR-92a-2) encoded by two different loci. ' ${ }^{\mathrm{miR}-19}$ includes two identical sequences (miR-19b-1 and miR-19b-2) encoded by two different loci. 
Table 2 Expression change of senescence-associated miRNAs in IMR90 cells treated with DEM or etoposide

\begin{tabular}{|c|c|c|c|c|c|c|}
\hline \multirow[b]{2}{*}{ miRNA } & \multicolumn{3}{|c|}{ DEM } & \multicolumn{3}{|c|}{ Etoposide } \\
\hline & Fold variation $^{a}$ & S.D. & $P$-value & Fold variation ${ }^{a}$ & S.D. & $P$-value \\
\hline miR-486-5p & 1.55 & 0.118 & 0.0018 & 3.22 & 0.07 & $<0.0001$ \\
\hline miR-210 & 1.69 & 0.12 & $<0.0001$ & 1.5 & 0.2 & 0.0001 \\
\hline miR-30e-5p & 3.25 & 0.57 & 0.0008 & 1.82 & 0.03 & $<0.0001$ \\
\hline miR-376a* & 2.87 & 0.89 & $<0.0001$ & 1.7 & 0.27 & 0.0023 \\
\hline miR-126* & 2.35 & 0.28 & 0.0004 & 2.05 & 0.15 & 0.0001 \\
\hline miR-494 & 2.14 & 0.2 & 0.0011 & 0.93 & 0.04 & 0.2652 \\
\hline miR-379 & 1.52 & 0.06 & 0.0001 & 1.39 & 0.08 & 0.0070 \\
\hline miR-654 & 2.29 & 0.53 & 0.0046 & 1.16 & 0.8 & 0.5177 \\
\hline$m i R-542-5 p$ & 1.128 & 0.14 & 0.5818 & 1.22 & 0.04 & 0.0224 \\
\hline miR-23b & 1.94 & 0.12 & 0.0002 & 1.42 & 0.18 & 0.0105 \\
\hline miR-134 & 1.52 & 0.12 & 0.0032 & 1.67 & 0.002 & $<0.0001$ \\
\hline miR-369-3p & 1.44 & 0.13 & 0.0036 & 1.61 & 0.03 & $<0.0001$ \\
\hline miR-656 & 1.26 & 0.06 & 0.0181 & 1.21 & 0.04 & 0.0137 \\
\hline miR-485-5p & 2.33 & 0.08 & 0.0001 & 1.36 & 0.11 & 0.0420 \\
\hline miR-20a & 0.419 & 0.002 & $<0.0001$ & 0.29 & 0.03 & $<0.0001$ \\
\hline miR-155 & 0.2 & 0.002 & $<0.0001$ & 0.32 & 0.014 & $<0.0001$ \\
\hline$m i R-17-5 p$ & 0.51 & 0.01 & $<0.0001$ & 0.43 & 0.04 & 0.0086 \\
\hline$m i R-199 b-5 p$ & 0.55 & 0.06 & 0.069 & 0.37 & 0.02 & $<0.0001$ \\
\hline$m i R-15 b$ & 0.33 & 0.016 & $<0.0001$ & 0.33 & 0.02 & 0.0013 \\
\hline miR-92 & 0.43 & 0.08 & 0.0018 & 0.37 & 0.01 & 0.0003 \\
\hline miR-296-5p & 0.72 & 0.005 & 0.0007 & 0.7 & 0.02 & 0.0059 \\
\hline miR-19b & 0.39 & 0.14 & 0.0125 & 0.33 & 0.04 & 0.0002 \\
\hline miR-93 & 0.45 & 0.02 & 0.0006 & 0.46 & 0.06 & 0.0009 \\
\hline miR-106b & 0.84 & 0.01 & $<0.0001$ & 0.65 & 0.07 & 0.0396 \\
\hline
\end{tabular}

aWith respect to untreated cells.

(Supplementary Figure 2). RNA was extracted from DEMand etoposide-treated IMR90 cells, and the expression of the 24 senescence-associated miRNAs was studied in triplicate by qRT-PCR. Results are reported in Table 2 . About $90 \%$ of the senescence-associated miRNAs were de-regulated (albeit with smaller fold changes than during replicative senescence) upon chemically induced senescence $(P<0.05)$. In detail, DEM-mediated senescence was associated with the upregulation (range: 1.26-3.25) of 13 of the 14 (with the exception of miR-542-5p) upregulated senescence-associated miRNAs $(P<0.05)$ (Table 2), whereas etoposide-mediated senescence was associated with the upregulation (range: 1.21-3.22) of 12 (with the exception of miR-654 and miR-494) of them $(P<0.05)$ (Table 2). Moreover, both DEM-treated (range: 0.84-0.2) as well as etoposide-treated (range: $0.7-0.29$ ) cells showed downregulation of all the 10 downregulated senescenceassociated miRNAs $(P<0.05)$ (Table 2$)$.

These findings indicate that DNA damage and oxidative stress alter the expression of senescence-associated miRNAs in IMR90 cells, thereby suggesting that DNA damage and oxidative stress, occurring during replicative senescence, may contribute to the altered expression of senescenceassociated miRNAs.

A set of miRNAs promotes a senescent phenotype in IMR90 cells. We sought to establish whether upregulation of senescence-associated miRNAs was causally related to senescence. Thus, the nine highest ranked upregulated miRNAs (Table 1) were adoptively upregulated (in the form of synthetic pre-miRNAs) by electroporation in young PDL33 IMR90 cells and their capability of inducing senescenceassociated markers was assessed. Negative control was represented by scrambled pre-miRNA-transfected PDL33 IMR90 cells. Positive control was represented by PDL55 (or 58) IMR90 cells. Seven out of the nine miRNAs induced a significant $(P<0.05)$ increase of SAHF-positive nuclei (range: $10-24 \%$ of the cells) detected by both DAPI staining and $3 \mathrm{meH} 3 \mathrm{~K} 9$ immunofluorescence with respect to the scrambled control (Figures $1 \mathrm{a}$ and $\mathrm{b}$ ). This was accompanied by a 4 - to 18-fold increase of SA- $\beta$-gal-positive cells $(P<0.05)$ (Figure 1c). miR-654 did not cause any significant increase of SAHF-positive and SA- $\beta$-gal-positive cells; instead, we noted a robust cytotoxic effect exerted by this miRNA, assessed as percentage of dead cells upon transfection (data not shown). miR-654 was not studied further.

Senescent cells are arrested in the $\mathrm{G}_{1}$ phase of the cell cycle. Therefore, we tested whether expression of the highest ranked upregulated miRNAs was also able to reduce BrdU incorporation into newly synthesized DNA. Electroporation of five of them (miR-210, miR-376a*, miR-486-5p, miR-494, and miR-542-5p) in PDL33 IMR90 cells caused a significant $(P<0.001)$ reduction (2- to 3 -fold) of BrdU incorporation (Figure 2a). Moreover, these five miRNAs also caused a parallel reduction (approximately 30-60\%) in cell number $(P>0.05)$ (Figure $2 \mathrm{~b}$ ). miR-126* did not exert any detectable effect, and miR-379 and miR-30e-5p caused only negligible reduction of BrdU incorporation and cell proliferation $(P<0.05)$ (Figures $2 \mathrm{a}$ and b). Negative control was represented by scrambled miRNA-transfected cells (Figures $2 \mathrm{a}$ and $\mathrm{b})$.

Altogether, these findings show that seven senescenceinduced miRNAs are able to induce SAHF/SA- $\beta$-gal positivity, with five of them (miR-210, miR-376a*, miR-486-5p, miR-494, and miR-542-5p) causing a robust reduction of cell proliferation and DNA synthesis rate.

miR-210, miR-376a*, miR-486-5p, miR-494, and miR-542$5 p$ enhance DNA damage. DNA damage and DDR are 


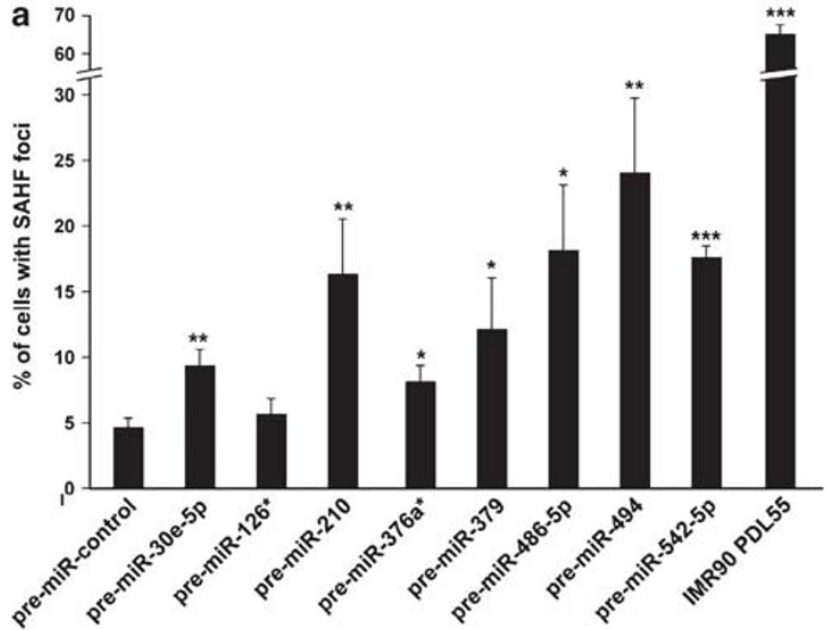

b
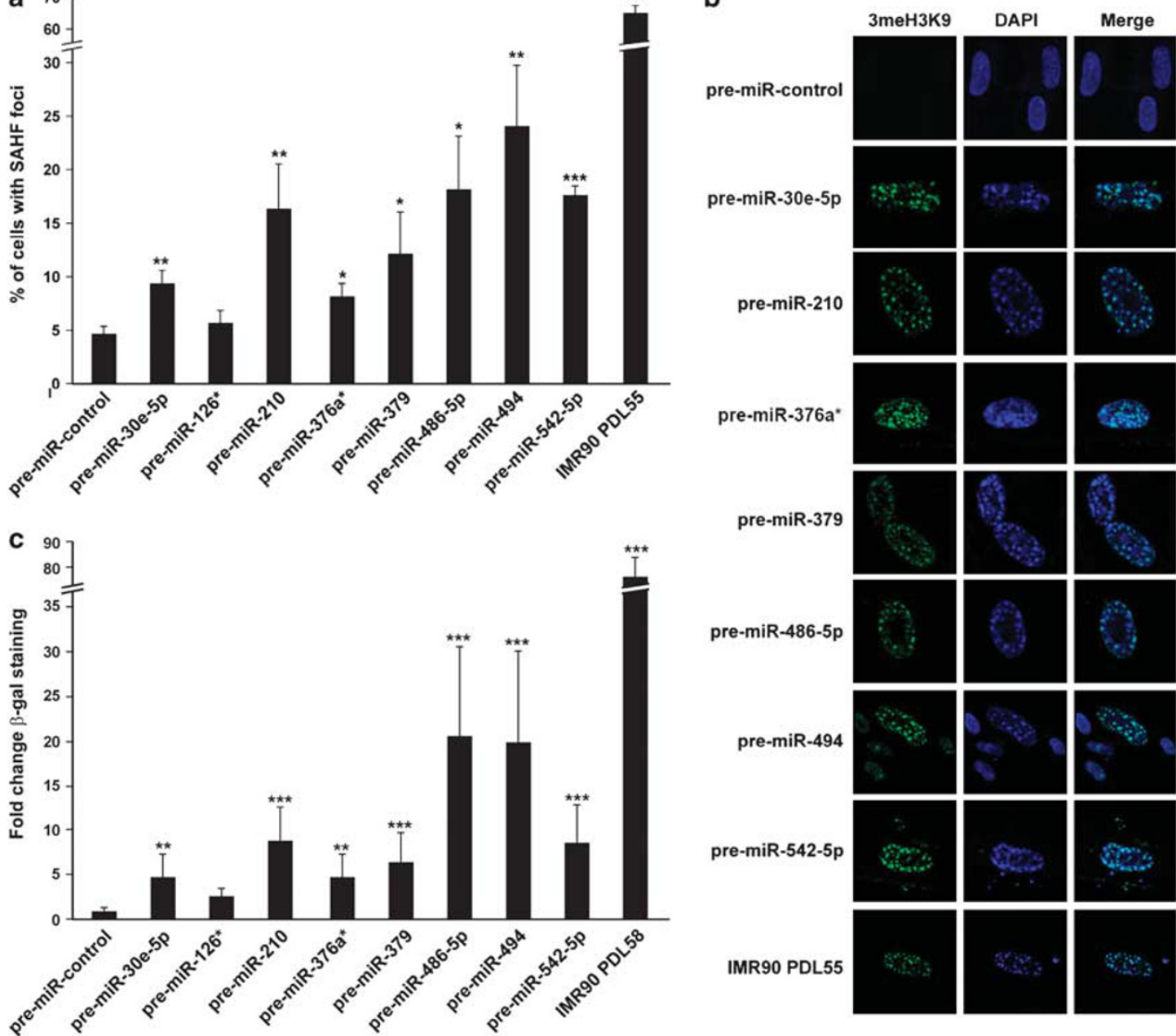

Figure 1 Senescence-associated miRNAs induce SAHFs and SA- $\beta$-gal. The nine highest ranked upregulated pre-miRNAs were electroporated in triplicate in PDL33 IMR90 cells. After 7 days cells were fixed and examined by immunofluorescence for $\alpha$-trimethyl-Lys 9 Histone (SAHF) or counterstained with DAPI (a and $\mathbf{b}$ ), and stained for SA- $\beta$-gal (c). ( $(\mathbf{a}$ and $\mathbf{b})$ Counts of at least 300 cells were averaged and expressed as percent of cells \pm S.D. with SAHF foci. Representative pictures are shown in panel $\mathbf{b}$. (c) Cells were stained for SA- $\beta$-gal. Counts of at least 300 cells were averaged and expressed as fold change \pm S.D. with respect to the scrambled control. Experimental points with ${ }^{*} P<0.05,{ }^{* *} P<0.01$, or ${ }^{* *} P<0.001$ respectively

involved in senescence. ${ }^{5}$ Therefore, we asked whether the five senescence-associated miRNAs, that were able to reduce proliferation rate and promote a senescent phenotype, were able to induce DNA damage. To this aim, we used neutral COMET assay to monitor double-strand DNA breaks. Etoposide-treated and scrambled miRNAtransfected cells were used as positive or negative controls, respectively. Tail moments were calculated and percentages of nuclei showing different tail moments were determined. The results shown in Figure 3 demonstrate that all the five miRNAs, but not the scrambled control, caused significant $(P<0.05)$ increase in the percentage of cells with tail moment $>4$, consistent with induction of double-strand DNA breaks (Figure 3).

Finally, we evaluated whether adoptive miRNA expression induced the formation of $\gamma \mathrm{H} 2 \mathrm{AX}$ foci in cell nuclei. Senescent cells and etoposide-treated cells were used as positive controls, whereas scrambled miRNA-transfected cells were used as negative controls. Figure 4 shows that miR-210 and
miR-494, but not the scrambled control, induced the formation of $\gamma \mathrm{H} 2 \mathrm{AX}$ foci in more than $25 \%$ of cell nuclei $(P<0.01)$. miR$376 a^{*}$, miR-486-5p, and miR-542-5p increased the number of $\gamma \mathrm{H} 2 \mathrm{AX}$ foci-positive nuclei only modestly $(P<0.05)$ (Figure 4$)$.

These findings suggest that senescence-associated miRNAs facilitate the establishment of a senescent state by promoting DNA damage and in some cases through increase of a DDR marker.

Role of oxidative stress and the mTOR-signaling pathway in miRNA-induced cellular senescence. Increased intracellular oxygen radicals can elicit DDR and senescence. ${ }^{5}$ Therefore, we investigated whether overexpression of senescence-associated miRNAs was able to cause intracellular accumulation of superoxide anion, by flow cytometry upon cell staining using the oxidation-sensitive DHE fluorescent probe. The results reported in Figure 5 demonstrate that overexpression of all five miRNAs (miR-210, miR-376a*, miR-486-5p, miR-494, and miR-542-5p) caused a significant 

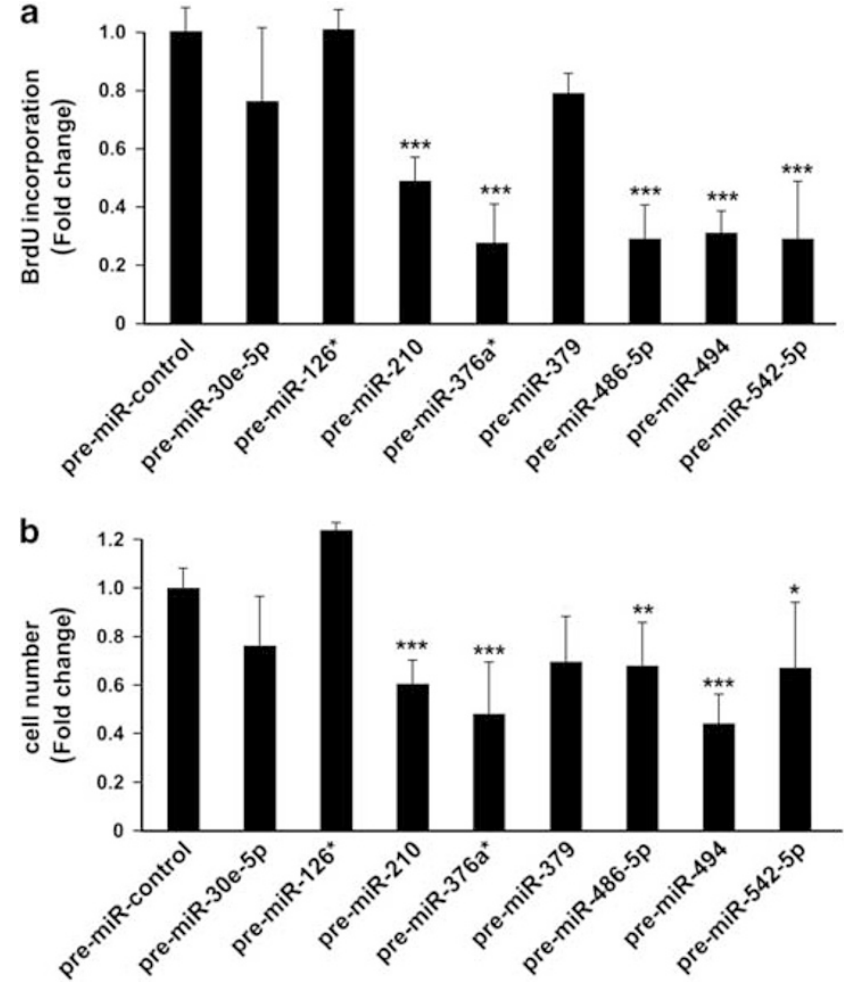

Figure 2 Senescence-associated miRNAs reduce cell proliferation. The nine highest ranked upregulated pre-miRNAs were electroporated in triplicate in PDL33 IMR90 cells. (a) After $48 \mathrm{~h}$ cells were incubated with BrdU for $24 \mathrm{~h}$ and fixed. Coverslips were incubated with an anti-BrdU and secondary fluorescein-conjugated antibody, counterstained with Hoechst-33258, and counted by immunofluorescence. Counts of at least 300 cells were averaged and expressed as fold change \pm S.D. with respect to the scrambled control. (b) After $96 \mathrm{~h}$ cell numbers were counted and expressed as fold change \pm S.D. with respect to the scrambled control. Experimental points with ${ }^{*} P<0.05,{ }^{* \star} P<0.01$, or ${ }^{\star \star *} P<0.001$ respectively
$(P<0.05)$ increase in DHE-positive cells with respect to the scrambled control. Of note, miR-210 was previously reported to induce the generation of ROS. ${ }^{18,19}$ Furthermore, it appears of interest that miR-494, which caused the highest levels of ROS generation, also caused the highest level of DNA damage as shown by the COMET assay (Figure 3 ).

The mammalian target of rapamycin (mTOR) pathway was demonstrated to drive senescence in cells treated with several factors, including oxidative agents. ${ }^{20-22}$ Thus, initially, we asked whether miRNA transfection caused any change in mTOR pathway activation, by measuring the phosphorylation of the ribosomal S6 protein (S6); S6 is phosphorylated by p70-S6 kinase (p70S6K), which, in turn, is activated by mTOR. ${ }^{23}$ IMR90 cells featured high levels of S6 phosphorylation, and adoptive overexpression of any of the five miRNAs (miR-210, miR-376a*, miR-486-5p, miR-494, and miR-542$5 p)$ did not cause detectable change in the levels of phosphoS6 (Supplementary Figure 3). Then, we tested whether inhibition of mTOR by the macrolide antibiotic rapamycin affected cell senescence (SA- $\beta$-gal) induced by miRNA transfection. Treatment with rapamycin caused a detectable reduction of SA- $\beta$-gal-positive cells upon transfection with the five miRNAs, a reduction that was statistically significant $(P<0.05)$ in the case of four of them (Table 3$)$. Rapamycin's effects on SA- $\beta$-gal stain were not accompanied by significant reduction of $\gamma-\mathrm{H} 2 \mathrm{AX}$ foci (Table 3 ).

Altogether, these findings suggest that the senescent phenotype induced by miR-210, miR-376a*, miR-494, and miR-542-5p depends on the activity of the mTOR pathway.

\section{Discussion}

Here we have identified a set of 24 miRNAs either up- or downregulated in senescent human diploid fibroblasts.

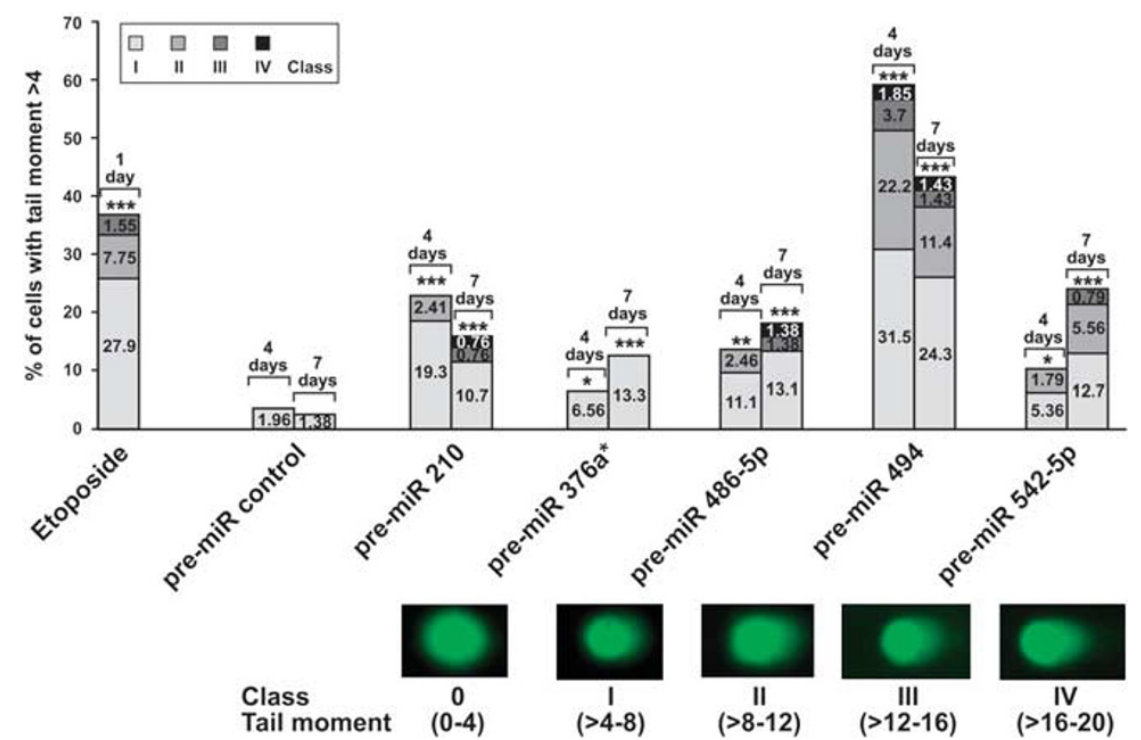

Figure 3 Senescence-associated miRNAs induce DNA damage. The indicated pre-miRNAs were electroporated in PDL33 IMR90 cells. After 4 or 7 days cells were harvested and comet tails were generated by electrophoresis. Slides were stained with SYBER Green and DNA migration was analyzed by fluorescence microscopy. Tail moment was determined and nuclei were divided in classes based on the value of the tail moment (as shown in the representative images of cells transfected with pre-miR-494 at the bottom). The percentage of damaged cells for each class is indicated within the bar graphs. A minimum of 100 cells per experiment were analyzed. Experimental points with ${ }^{\star} P<0.05,{ }^{* *} P<0.01$, or ${ }^{\star * \star} P<0.001$ respectively 


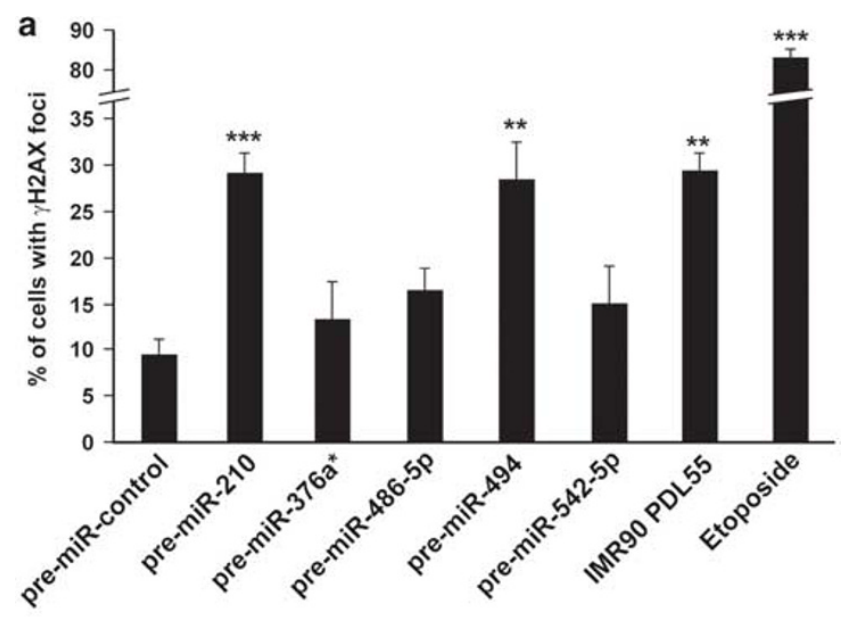

b
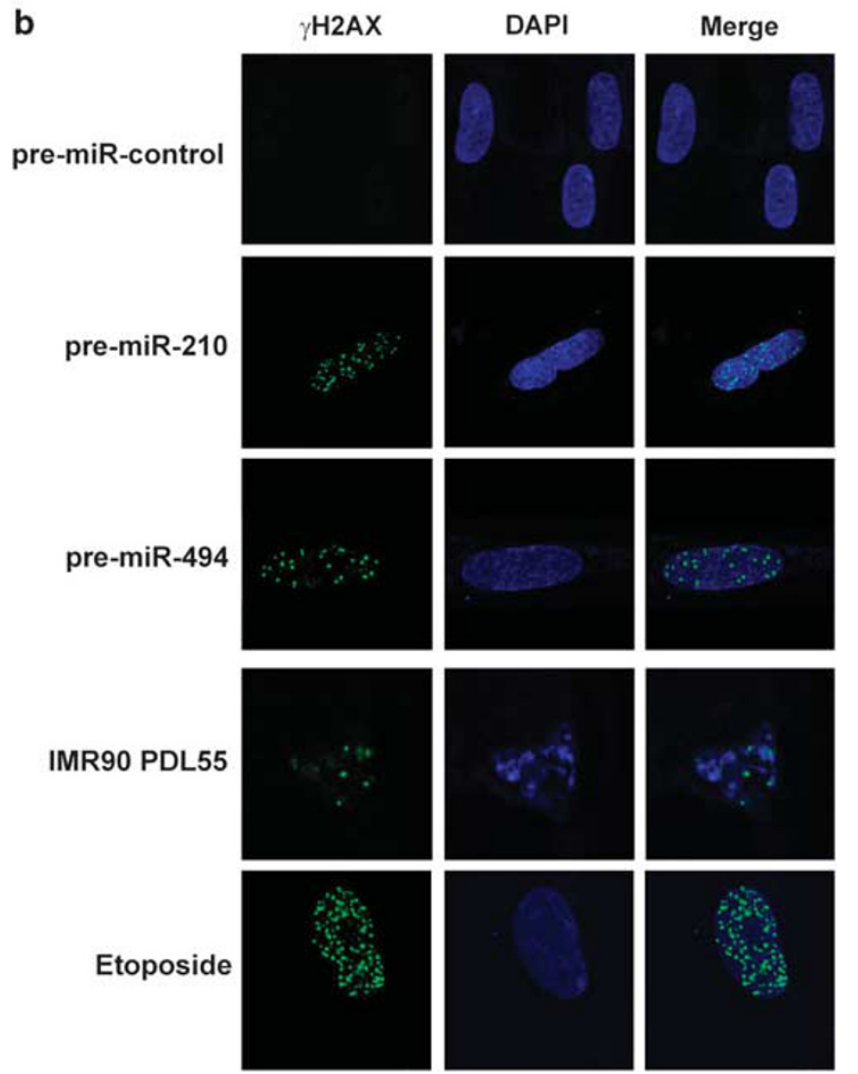

Figure 4 Senescence-associated miRNAs induce $\gamma \mathrm{H} 2 \mathrm{AX}$ foci. The indicated pre-miRNAs were electroporated in triplicate in PDL33 IMR90 cells. After 7 days cells were fixed and examined by immunofluorescence for $\alpha$-H2AX phosphorylated on Ser139 $(\gamma \mathrm{H} 2 \mathrm{AX})$. Coverslips were washed and incubated with an Alexa-488 goat anti-rabbit antibody and counterstained with DAPI. Counts of at least 300 cells were averaged and expressed as percent of cells \pm S.D. with $\gamma \mathrm{H} 2 \mathrm{AX}$ foci. Representative images are shown in panel $\mathbf{b}$. Experimental points in panel (a) are indicated with with ${ }^{* *} P<0.01$, or ${ }^{* * *} P<0.001$ respectively

Of note, 8 (chromosome 14q32.31) upregulated and 6 (4 on chromosome 13q31.3 and 2 on chromosome $7 q 22.1$ ) downregulated miRNAs mapped in specific chromosomal clusters, suggesting that their expression might be coregulated. We have also shown that the expression of most of the senescence-associated miRNAs was modified in DEM-

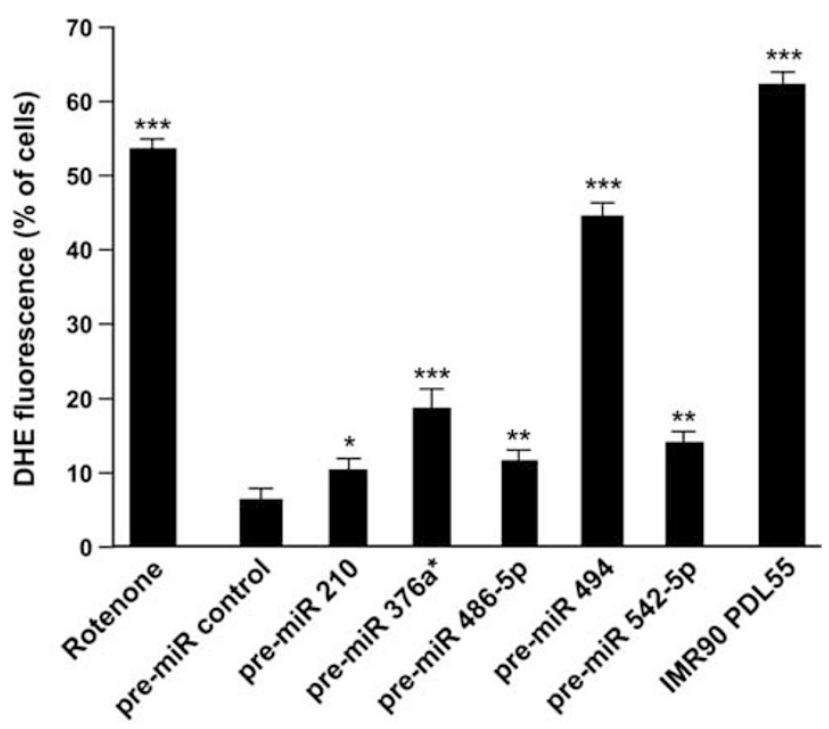

Figure 5 Senescence-associated miRNAs lead to oxidative stress. IMR90 cells (PDL33) were electroporated with the indicated pre-miRNAs. After $72 \mathrm{~h}$ cells were incubated with $0.1 \mu \mathrm{M}$ DHE at $37^{\circ} \mathrm{C}$ for $30 \mathrm{~min}$. Intracellular fluorescence was examined by flow cytometry. Fluorescence intensity of 10000 cells was analyzed in triplicate and expressed as percentage of stained cells \pm S.D. As positive controls, PDL33 cells treated with the mitochondrial inhibitor rotenone $(5 \mu \mathrm{M}$ for $2 \mathrm{~h})$ and senescent PDL55 cells were used. Experimental points with ${ }^{\star} P<0.05$, ${ }^{\star *} P<0.01$, or ${ }^{\star \star *} P<0.001$ respectively

Table 3 Effects of mTOR inhibition by rapamycin on miRNA-induced senescence and DDR

\begin{tabular}{lcccc}
\hline & $\begin{array}{c}\text { SA- } \boldsymbol{\beta} \text {-gal } \\
\text { staining } \\
\text { upon } \\
\text { rapamycin } \\
\text { (\% change) }\end{array}$ & $\boldsymbol{P}$-value & $\begin{array}{c}\text { (1) } \\
\text { staining } \\
\text { upon } \\
\text { rapamycin } \\
\text { (\% change) }\end{array}$ & \\
Pre-miRNA & $\boldsymbol{P}$-value \\
\hline Pre-miR-control & -16.2 & 0.4711 & +2.56 & 0.8520 \\
Pre-miR-210 & -43 & 0.0498 & -2.34 & 0.0808 \\
Pre-miR-376a* & -37.6 & 0.0052 & -2.75 & 0.2354 \\
Pre-miR-486-5p & -15.3 & 0.2526 & +7.69 & 0.5734 \\
Pre-miR-494 & -46.2 & 0.0050 & -6.59 & $>0.999$ \\
Pre-miR-542-5p & -38.9 & 0.0262 & +6.38 & 0.9360 \\
\hline
\end{tabular}

${ }^{\text {a }}$ Starting $24 \mathrm{~h}$ after transfection with the indicated miRNAs, cells were treated for 3 days with $10 \mathrm{nM}$ rapamycin or vehicle, and then stained for SA- $\beta$-gal. Counts of at least 300 cells were averaged and expressed as percentage of SA- $\beta$-gal-stained cells. Reduction in the percentage of positive cells upon rapamycin treatment is reported. ${ }^{b} S t a r t i n g ~ 24 \mathrm{~h}$ after transfection with the indicated miRNAs, cells were treated for 3 days with $10 \mathrm{nM}$ rapamycin or vehicle, and then examined by immunofluorescence for $\gamma \mathrm{H} 2 \mathrm{AX}$. Counts of at least 300 cells were averaged and expressed as percent of cells with $\gamma \mathrm{H} 2 \mathrm{AX}$ foci. Change in the percentage of positive cells upon rapamycin treatment is reported.

or etoposide-induced senescence, in the same direction as in replicative senescence. This suggests that oxidative stress and DNA damage are among the factors that mediate senescence-associated miRNA expression changes. However, the effects of DEM and etoposide were smaller than those measured upon replicative senescence, suggest- 
ing that either the contribution of other factors or the conditions of chemical treatments were not sufficient to achieve a robust change of miRNA expression.

We have also shown that adoptive expression of five upregulated miRNAs (miR-210, miR-376a*, miR-486-5p, miR-494, and miR-542-5p) induced a senescent phenotype (with SAHFs, SA- $\beta$-gal staining, and reduced cell proliferation). Overexpression of these five miRNAs was also associated with double-strand DNA breaks in a neutral COMET assay and, for two of them (miR-210 and miR-494), appearance of a DDR marker $(\gamma \mathrm{H} 2 \mathrm{AX}$ foci). Thus, overexpression of the five miRNAs favored DNA damage, and not solely DDR ( $\gamma \mathrm{H} 2 \mathrm{AX})$, which can also occur in the absence of DNA damage (pseudo-DDR). ${ }^{24}$ Finally, we showed that the mTOR pathway was an essential driver of senescence induced by senescence-associated miRNAs as SA- $\beta$-gal staining was significantly reduced by treatment with rapamycin. ${ }^{20-22}$ Of note, our preliminary observations, by studying the expression levels of the senescence-inducing miRNAs in human skin broblasts from young (age 17-25) or old (age 89-94) donors, show that the highest expression levels of miR-376a* and miR-494 were found in one old donor cell line, and expression of miR-486-5p was on average two-fold higher in old with respect to young cells.

Overall, these findings suggest a model whereby DNA damage and oxidative stress, which occur during replicative senescence, stimulate the expression of a set of miRNAs, which, in turn, feedback positively to DNA damage and oxidative stress, thereby sustaining the senescent phenotype (Supplementary Figure 4). The mechanisms through which the upregulated miRNAs provoke these effects will deserve further investigation. However, the model is supported by the observation that overexpression of the senescence-associated miRNAs caused an accumulation of superoxide anion and by the fact that some known targets of senescenceassociated miRNAs are involved in DNA damage, oxidative stress, cell-cycle control, and apoptosis. For instance, miR-210 was found to activate the generation of ROS by targeting subunits of the electron transport chain (ETC) complexes I and II. ${ }^{18}$ Other potential targets of miR-210 in the same pathway included ISCU (iron-sulfur cluster scaffold homolog) and COX10 (cytochrome-c oxidase assembly protein), two factors of the mitochondrial electron transport chain and the tricarboxylic acid cycle. ${ }^{19}$ In addition, forced expression of miR-210 decreased endogenous levels of RAD52, a key factor in homology-dependent DNA repair (HR). ${ }^{25}$ Similarly, miRNA-494 was recently found to be localized to mitochondria, where it may target genes involved in ATP synthesis-coupled electron transport. ${ }^{26}$ Of note, miR-210 also suppresses cell-cycle progression by targeting $\mathrm{E}_{2} \mathrm{F3}^{27}$ and fibroblast growth factor receptor-like-1 (FGFRL1). ${ }^{28}$ miR-376a* was reported to repress phosphoribosyl pyrophosphate synthetase-1 (PRPS1) involved in DNA/ RNA synthesis as well as threonine and tyrosine kinase (TTK) essential for mitosis checkpoint. ${ }^{29}$ miR-494 was upregulated in cells exposed to the DNA-damaging agent benzo[a]pyrene $(\mathrm{B}[\mathrm{a}] \mathrm{P})$ and was found to restrain cell cycle through CDK6 downregulation. ${ }^{30}$ Finally, miR-542-5p was upregulated in senescent BJ cells ${ }^{31}$ and downregulated in neuroblastoma and other solid tumors. ${ }^{32}$
Though not investigated in our study, it is tempting to speculate that also senescence-downregulated miRNAs contribute to the execution of the senescence program (Supplementary Figure 4). In our study, several miRNAs of the miR-17 family (miR-17-5p, miR-20a, miR-106b, and miR-93) and miR-155 were downregulated both in replicative and chemically induced senescence. Noteworthy, the miR-17 family ${ }^{33}$ and miR-155 $5^{12}$ have already been involved in cellular senescence. Hackl et al. ${ }^{11}$ reported that miRNAs of the miR17 family were downregulated in replicative senescence and in organismal aging models, their decrease correlating with increased levels of p21WAF1. miRNAs of the miR-17 family (miR-17-92 cluster) were also upregulated in cancer ${ }^{34-37}$ and, by targeting p21WAF1, they were able to confer resistance to RAS-induced senescence. ${ }^{38}$ On the other hand, miR- 155 was downregulated in senescent WI-38 cells, causing increased levels of TP53INP1, a protein acting in the TP53 growth arrest pathway. ${ }^{12}$ Another link between miR-155 and cell proliferation comes from recent data showing that WEE1 (WEE1 homolog-Schizosaccharomyces pombe), a kinase that blocks cell-cycle progression, is a target of this miRNA. ${ }^{39}$

In conclusion, we have identified a set of miRNAs, which, being modulated by senescence-mediating conditions, and being able to either facilitate DNA damage or regulate the expression of several senescence mediators, may represent critical components of the cellular senescence program.

\section{Materials and Methods}

Cell cultures. Normal primary human fibroblasts IMR90 were obtained from American Type Culture Collection (Manassas, VA, USA). IMR90 were grown in Dulbecco's modified Eagle's medium (DMEM) (Invitrogen, Groningen, The Netherlands) supplemented with $10 \%(\mathrm{v} / \mathrm{v})$ fetal bovine serum and $1 \%$ penicillin/streptomycin (Invitrogen). Cultures were maintained at $37^{\circ} \mathrm{C}$ in a $5 \% \mathrm{CO}_{2^{-}}$ humidified atmosphere. The population doubling level (PDL) was calculated by using the formula $\triangle \mathrm{PDL}=\log \left(n_{h} / n_{i}\right) / \log 2$, where $n_{i}$ is the initial number of cells and $n_{h}$ is the final number of cells at each passage. The cells were used at $33 \mathrm{PDL}$ or 58 PDL. More than $70 \%$ of PDL58 cells were positive for SA- $\beta$-gal. SA- $\beta$-gal was assayed according to Dimri et al. ${ }^{14}$ Briefly, cells were washed twice with PBS, fixed with $2 \%$ formaldehyde and $0.2 \%$ glutaraldehyde in PBS, and washed twice in PBS. Then, cells were stained overnight in X-gal staining solution $(1 \mathrm{mg} / \mathrm{ml} \mathrm{X-gal,} 40 \mathrm{mM}$ citric acid/sodium phosphate $(\mathrm{pH} 6.0), 5 \mathrm{mM}$ potassium ferricyanide, $5 \mathrm{mM}$ potassium ferrocyanide, $150 \mathrm{mM} \mathrm{NaCl}, 2 \mathrm{mM} \mathrm{MgCl}$ ).

Diethylmaleate (DEM) was purchased from Sigma Chemical Co. (St. Louis, MO, USA) and used at a final concentration of $100 \mu \mathrm{M}$ in complete medium. ${ }^{9}$ Etoposide (VP-16; Calbiochem, La Jolla, CA, USA) was used at $20 \mu \mathrm{M} .{ }^{15}$ Rapamycin was obtained from Merck Chemicals Ltd (Nottingham, UK) and used at $10 \mathrm{nM}$. Rotenone (Sigma Chemical Co.) was used at $5 \mu \mathrm{M}$.

miRNA profiling. Total RNA was isolated using the mirVana MiRNA isolation kit (Ambion, Austin, TX, USA) according to the manufacturer's instructions. The RNA was quantified by Nanodrop (Thermo Scientific, Wilmington, DE, USA) and RNA integrity was analyzed by using the 2100 Bioanalyzer (Agilent Technologies, Waldbronn, Germany). Global miRNA expression analysis was performed by using TaqMan Low Density Arrays (TLDA)/Human microRNA Panel v1.0 (Applied Biosystems, Foster City, CA, USA). This technology detects mature miRNAs from the Sanger miRBase database. Each card/panel contains a primerprobe set for 365 human miRNAs. Briefly, for each sample, $100 \mathrm{ng}$ of total RNA was converted into CDNA by multiplex reverse transcription (RT) using a primer pool from the Taqman array kit (Applied Biosystems), according to the manufacturer's instructions. Then, miRNA levels were measured by quantitative real-time PCR (qRT-PCR) using the 7900HT Sequence Detection System (Applied Biosystems). $C_{t}$ values were determined by using the automatic threshold in $R Q$ manager v1.1 analysis software (Applied Biosystems). Data were analyzed by using the Sequence Detection System software (v. 2.3) (Applied Biosystems). The endogenous control 
RNU48 was used to normalize the relative expression of each miRNA using the $\Delta \Delta C_{t}$ method (Applied Biosystems User Bulletin No. 2-P/N 4303859).

Real-time PCR. Total RNA was isolated by using the mirVana MiRNA isolation kit (Ambion) according to the manufacturer's instructions. The RNA was quantified by Nanodrop (Thermo Scientific) and RNA integrity was analyzed by the 2100 Bioanalyzer (Agilent Technologies). The TaqMan MiRNA Assay kit (Applied Biosystems) was used to detect the expression of mature miRNAs. Briefly, $100 \mathrm{ng}$ of total RNA was reverse-transcribed (RT) at $16^{\circ} \mathrm{C}$ for $30 \mathrm{~min}, 42^{\circ} \mathrm{C}$ for $30 \mathrm{~min}$, and $85^{\circ} \mathrm{C}$ for $5 \mathrm{~min}$ in a $15-\mu \mathrm{l}$ reaction volume. $2-\mu \mathrm{l}$ volume of the RT product was used for PCR in a final volume of $20 \mu \mathrm{l}$. The PCR started with an initial denaturation step at $90^{\circ} \mathrm{C}$ for $10 \mathrm{~min}$, followed by 40 cycles of $95^{\circ} \mathrm{C}$ for $15 \mathrm{~s}$ and $60^{\circ} \mathrm{C}$ for $1 \mathrm{~min}$. Small nucleolar RNA RNU6 (Applied Biosystems) was used for normalization. PCRs were performed in triplicate and fold changes were calculated by the following formula: $2^{\left.- \text {(sample-1 } 1 \Delta C_{1}-\text { sample-2 } 2 \Delta C_{1}\right)}$, where $\Delta C_{t}$ is the difference between the amplification fluorescent thresholds of the miRNA of interest and the RNA of RNU6.

Cell electroporation. PDL33 IMR90 cells were electroporated by using Microporator MP100 (EuroClone, Milano, Italy) following the manufacturer's instructions. Briefly, $1 \times 10^{6} \mathrm{cells}$ were electroporated in $100 \mu \mathrm{l}$ of suspension buffer (Neon transfection kit; Invitrogen) using $100 \mathrm{nM}$ pre-miRNA (Ambion) according to the manufacturer's protocol adapted to IMR90 cells (pulse voltage: $1400 \mathrm{~V}$ for $10 \mathrm{~ms}$, three pulses). Electroporation efficiency was tested by using the Cy3-labeled negative control (Ambion) according to the manufacturer's protocol and was approximately of $75 \%$. After electroporation, cells were seeded and viable cells were counted at $96 \mathrm{~h}$.

BrdU assay. For BrdU (5-bromo-2-deoxyuridine) incorporation assay, electroporated cells were seeded on glass coverslips. Forty-eight hours after transfection, cells were incubated for $24 \mathrm{~h}$ with $\mathrm{BrdU}(10 \mu \mathrm{M})$ and fixed. Coverslips were incubated with an anti-BrdU and secondary fluorescein-conjugated antibody. Coverslips were counterstained with Hoechst-33258, rinsed, and mounted in Movio on glass slides. The fluorescent signal was visualized with an epifluorescent microscope (Axiovert 2; Zeiss, Gottingen, Germany), interfaced with the image analyzer software KS300.

Immunofluorescence. For indirect immunofluorescence, cells were fixed in $4 \%$ paraformaldehyde and permeabilized with $0.2 \%$ Triton X-100 ( 5 min on ice), and then incubated with $\alpha-\mathrm{H} 2 \mathrm{AX}$ phosphorylated on Ser139 ( $\gamma \mathrm{H} 2 \mathrm{AX})$ (R\&D Systems, Minneapolis, MN, USA) or $\alpha$-trimethyl-Lys9 Histone H3 (Lake Placid Biologicals, Lake Placid, NY, USA) for $1 \mathrm{~h}$ at room temperature. Coverslips were washed and incubated with an Alexa-488 goat anti-rabbit antibody (Invitrogen) for $30 \mathrm{~min}$ at room temperature. After $5 \mathrm{~min}$ of DAPI counterstaining, coverslips were mounted and observed with a Zeiss LSM 510 META confocal microscope (Carl Zeiss, Thornwood, NY, USA). At least 300 cells were counted in triplicate experiments.

COMET assay. The neutral comet assay was performed according to the manufacturer's recommendations (Trevigen, Gaithersburg, MD, USA). ${ }^{16}$ Slides were incubated for $30 \mathrm{~min}$ in lysis buffer and for $40 \mathrm{~min}$ in alkaline solution $(\mathrm{pH}>13)$, and comet tails were generated by 15 -min electrophoresis in TBE buffer at $20 \mathrm{~V}$, at $4{ }^{\circ} \mathrm{C}$. The slides were stained with SYBER Green and DNA migration was analyzed by fluorescence microscopy (Leica DMS 4000B). Tail moment (TM), defined as the product of the tail length and the fraction of total DNA in the tail $(T M=$ tail length $\times \%$ of DNA in the tail), was determined by using the software 'Comet Assay II' (Perceptive Instruments, Suffolk, UK). A minimum of 100 cells per experiment were analyzed. All experiments were performed in triplicate.

ROS measurement. Reactive oxygen species (ROS) were measured by using the oxidation-sensitive fluorescent probe dihydroethidium (DHE; Sigma Chemical), according to a published procedure. ${ }^{17}$ Briefly, $72 \mathrm{~h}$ after miRNA transfection, cells were incubated for $30 \mathrm{~min}$ at $37^{\circ} \mathrm{C}$ with $0.1 \mu \mathrm{M}$ DHE, harvested, and resuspended in PBS. To determine fluorescence due to formation of superoxide anion, the cell suspension was subjected to flow-cytometric analysis using a Becton Dickinson FACSCalibur instrument (Becton Dickinson, San Josè, CA, USA), after excitation at $488 \mathrm{~nm}$ (FL-2 channel). Results from at least 10000 cells from each sample were analyzed in triplicate using the CellQuest 3.2.1 software (Becton Dickinson).
Statistical analysis. Statistical analyses were performed by using the GraphPad InStat software program (version 3.06.3, San Diego, CA, USA). All $P$-values were two-sided and differences were significant when $P<0.05$.

\section{Conflict of Interest}

The authors declare no conflict of interest.

Acknowledgements. We thank Professor T Russo for critical reading of the manuscript and Dr. F Merolla for help with statistical analysis. This study was supported by the Ministero dell'Università e della Ricerca Scientifica e Tecnologica (MiUR PRIN 2007, MiUR-PS35-126/IND, MiUR MERIT RBNE08HWLZ_004), Regione Campania LR 5/2003, Associazione Italiana per la Ricerca sul Cancro (AIRC), Italian Ministero della Salute, and Fondazione SDN per la Ricerca e l'Alta Formazione in Diagnostica Nucleare.

1. Hayflick L, Moorhead PS. The serial cultivation of human diploid cell strains. Exp Cell Res 1961; 25: 585-621.

2. Collado M. Serrano M. Senescence in tumours: evidence from mice and humans. Nat Rev Cancer 2010; 10: 51-57.

3. Collado M, Blasco MA, Serrano M. Cellular senescence in cancer and aging. Cell 2007; 130: 223-233.

4. Blasco MA. Telomeres and human disease: ageing, cancer and beyond. Nat Rev Genet 2005; 6: 611-622.

5. d'Adda di Fagagna F. Living on a break: cellular senescence as a DNA-damage response. Nat Rev Cancer 2008; 8: 512-522.

6. Campisi J, d'Adda di Fagagna F. Cellular senescence: when bad things happen to good cells. Nat Rev Mol Cell Biol 2007; 8: 729-740.

7. Coppé JP, Desprez PY, Krtolica A, Campisi J. The senescence-associated secretory phenotype: the dark side of tumor suppression. Annu Rev Pathol 2010; 5: 99-118.

8. Fridman AL, Tainsky MA. Critical pathways in cellular senescence and immortalization revealed by gene expression profiling. Oncogene 2008; 27: 5975-5987.

9. Faraonio R, Pane F, Intrieri M, Russo T, Cimino F. In vitro acquired cellular senescence and aging-specific phenotype can be distinguished on the basis of specific mRNA expression. Cell Death Differ 2002; 9: 862-864.

10. Garzon R, Calin GA, Croce CM. MicroRNAs in cancer. Annu Rev Med 2009; 60: 167-179.

11. Hackl M, Brunner S, Fortschegger K, Schreiner C, Micutkova L, Mück C et al. miR-17, miR-19b, miR-20a and miR-106a are downregulated in human aging. Aging Cell 2010; 9: 291-296.

12. Wang $Y$, Scheiber MN, Neumann C, Calin GA, Zhou D. MicroRNA regulation of ionizing radiation-induced premature senescence. Int J Radiat Oncol Biol Phys 2010; 81: 839-848.

13. Grillari J, Hackl M, Grillari-Voglauer R. miR-17-92 cluster: ups and downs in cancer and aging. Biogerontology 2010; 11: 501-506.

14. Dimri GP, Lee X, Basile G, Acosta M, Scott G, Roskelley C et al. A biomarker that identifies senescent human cells in culture and in aging skin in vivo. Proc Natl Acad Sci USA 1995 92: 9363-9367.

15. Te Poele RH, Okorokov AL, Jardine L, Cummings J, Joel SP. DNA damage is able to induce senescence in tumor cells in vitro and in vivo. Cancer Res 2002; 62: 1876-1883.

16. Minopoli G, Stante M, Napolitano F, Telese F, Aloia L, De Felice $M$ et al. Essential roles for Fe65 Alzheimer amyloid precursor-binding protein, in the cellular response to DNA damage. J Biol Chem 2007; 282: 831-835.

17. Trifunovic A, Hansson A, Wredenberg A, Rovio AT, Dufour E, Khvorostov I et al Somatic mtDNA mutations cause aging phenotypes without affecting reactive oxygen species production. Proc Natl Acad Sci USA 2005; 102: 17993-17998.

18. Puisségur MP, Mazure NM, Bertero T, Pradelli L, Grosso S, Robbe-Sermesant K et al. miR-210 is overexpressed in late stages of lung cancer and mediates mitochondria alterations associated with modulation of HIF-1 activity. Cell Death Differ 2011; 18 465-478.

19. Chen Z, Li Y, Zhang H, Huang P, Luthra R. Hypoxia-regulated microRNA-210 modulates mitochondrial function and decreases ISCU and COX10 expression. Oncogene 2010; 29 4362-4368.

20. Leontieva OV, Blagosklonny MV. DNA damaging agents and p53 do not cause senescence in quiescent cells, while consecutive re-activation of mTOR is associated with conversion to senescence. Aging (Albany NY) 2010; 2: 924-935.

21. Korotchkina LG, Leontieva OV, Bukreeva El, Demidenko ZN, Gudkov AV, Blagosklonny MV. The choice between p53-induced senescence and quiescence is determined in part by the mTOR pathway. Aging (Albany NY) 2010; 2: 344-352.

22. Demidenko ZN, Shtutman M, Blagosklonny MV. Pharmacologic inhibition of MEK and $\mathrm{PI}-3 \mathrm{~K}$ converges on the $\mathrm{mTOR} / \mathrm{S} 6$ pathway to decelerate cellular senescence. Cell Cycle 2009; 8: 1896-1900.

23. Hay N, Sonenberg N. Upstream and downstream of mTOR. Genes Dev 2004; 18 1926-1945. 
24. Pospelova TV, Demidenko ZN, Bukreeva El, Pospelov VA, Gudkov AV, Blagosklonny MV. Pseudo-DNA damage response in senescent cells. Cell Cycle 2009; 8: 4112-4118.

25. Crosby ME, Kulshreshtha R, Ivan M, Glazer PM. MicroRNA regulation of DNA repair gene expression in hypoxic stress. Cancer Res 2009; 69: 1221-1229.

26. Bandiera S, Ruberg S, Girard M, Cagnard N, Hanein S, Chretien D et al. Nuclear outsourcing of RNA interference components to human mitochondria. PLoS One 2011; 6: e20746.

27. Biswas S, Roy S, Banerjee J, Hussain SR, Khanna S, Meenakshisundaram G et al. Hypoxia inducible microRNA 210 attenuates keratinocyte proliferation and impairs closure in a murine model of ischemic wounds. Proc Natl Acad Sci USA 2010; 107: 6976-6981.

28. Tsuchiya S, Fujiwara T, Sato F, Shimada Y, Tanaka E, Sakai $Y$ et al. MicroRNA-210 regulates cancer cell proliferation through targeting fibroblast growth factor receptor-like 1 (FGFRL1). J Biol Chem 2011; 286: 420-428.

29. Kawahara $Y$, Zinshteyn $B$, Sethupathy $P$, lizasa $H$, Hatzigeorgiou AG, Nishikura $K$ Redirection of silencing targets by adenosine-to-inosine editing of miRNAs. Science 2007; 315: $1137-1140$.

30. Duan $\mathrm{H}$, Jiang Y, Zhang H, Wu Y. MiR-320 and miR-494 affect cell cycles of primary murine bronchial epithelial cells exposed to benzo[a]pyrene. Toxicol In Vitro 2010; 24: 928-935.

31. Bonifacio LN, Jarstfer MB. MiRNA profile associated with replicative senescence, extended cell culture, and ectopic telomerase expression in human foreskin fibroblasts. PLoS One 2010; 5: e12519.
32. Schulte JH, Marschall T, Martin M, Rosenstiel P, Mestdagh $\mathrm{P}$, Schlierf $\mathrm{S}$ et al. Deep sequencing reveals differential expression of microRNAs in favorable versus unfavorable neuroblastoma. Nucleic Acids Res 2010; 38: 5919-5928.

33. Mendell JT. miRiad roles for the miR-17-92 cluster in development and disease. Cell 2008 ; 133: 217-222.

34. He L, Thomson JM, Hemann MT, Hernando-Monge E, Mu D, Goodson S et al. A microRNA polycistron as a potential human oncogene. Nature 2005; 435: 828-833.

35. Volinia S, Calin GA, Liu CG, Ambs S, Cimmino A, Petrocca F et al. A microRNA expression signature of human solid tumors defines cancer gene targets. Proc Natl Acad Sci USA 2006; 103: 2257-2261.

36. O'Donnell KA, Wentzel EA, Zeller KI, Dang CV, Mendell JT. c-Myc-regulated microRNAs modulate E2F1 expression. Nature 2005; 435: 839-843.

37. Grillari J, Grillari-Voglauer R. Novel modulators of senescence, aging, and longevity: small non-coding RNAs enter the stage. Exp Gerontol 2010; 45: 302-311.

38. Hong L, Lai M, Chen M, Xie C, Liao R, Kang YJ et al. The miR-17-92 cluster of microRNAs confers tumorigenicity by inhibiting oncogene-induced senescence. Cancer Res 2010; 70: 8547-8557.

39. Tili E, Michaille JJ, Wernicke D, Alder H, Costinean S, Volinia S et al. Mutator activity induced by microRNA-155 (miR-155) links inflammation and cancer. Proc Natl Acad Sci USA 2011; 108: 4908-4913.

Supplementary Information accompanies the paper on Cell Death and Differentiation website (http://www.nature.com/cdd) 